\title{
Modeling the decay of entanglement for electron spin qubits in quantum dots
}

\author{
F Bodoky ${ }^{1}$, O Gühne ${ }^{2,3}$ and M Blaauboer ${ }^{1}$ \\ ${ }^{1}$ Kavli Institute of Nanoscience, Delft University of Technology, Lorentzweg 1, \\ 2628 CJ Delft, The Netherlands \\ ${ }^{2}$ Institut für Quantenoptik und Quanteninformation, Österreichische Akademie der \\ Wissenschaften, A-6020 Innsbruck, Austria \\ ${ }^{3}$ Institut für Theoretische Physik, Universität Innsbruck, Technikerstraße 25, \\ A-6020 Innsbruck, Austria \\ E-mail: f.bodoky@tnw.tudelft.nl, Otfried.Guehne@uibk.ac.at and m.blaauboer@tudelft.nl
}

Received 21 April 2009, in final form 1 July 2009

Published 1 September 2009

Online at stacks.iop.org/JPhysCM/21/395602

\begin{abstract}
We investigate the time evolution of entanglement under various models of decoherence: a general heuristic model based on local relaxation and dephasing times, and two microscopic models describing decoherence of electron spin qubits in quantum dots due to the hyperfine interaction with the nuclei. For each of the decoherence models, we investigate and compare how long the entanglement can be detected. We also introduce filtered witness operators, which extend the available detection time and investigate this detection time for various multipartite entangled states. By comparing the time required for detection with the time required for generation and manipulation of entanglement, we estimate for a range of different entangled states how many qubits can be entangled in a one-dimensional array of electron spin qubits.
\end{abstract}

(Some figures in this article are in colour only in the electronic version)

\section{Introduction}

Entanglement refers to non-classical correlations between two [1-4] or more [5, 6] quantum particles, and the creation of multiparticle entangled states constitutes a key step towards quantum computation [7]. In this work, we investigate the evolution of entangled states under different models of decoherence: a heuristic model with a broad range of applications, and two microscopic models specific for electron spin qubits in quantum dots [8]. We show how entanglement can be detected and how fast this needs to be done before the states become disentangled by decoherence. We also estimate for four different classes of multipartite entanglement which class survives the longest, and how many entangled qubits can be generated and detected with actual experimental means and currently known decoherence times.

We consider decoherence of a local nature, i.e. the qubits decohere in an uncorrelated way, as is the case in solid state nanosystems such as electron spin qubits in quantum dots [8], various superconducting qubits [9] and other solid state implementations ${ }^{4}$ : in these systems, the decoherence can

4 For a recent overview of more solid state qubit systems such as, for example, nitrogen-vacancy centers in diamond, see [10] and references therein. be characterized phenomenologically [11] by a relaxation time $T_{1}$ and a dephasing time $T_{2}$. The microscopic origin of the decoherence is still a matter of intensive research. In this paper we discuss some microscopic models for electron spin qubits in quantum dots and compare them with this heuristic model.

Our proposed means to analyze entanglement are socalled witness operators [12-16]: locally decomposable observables with a positive expectation value for all separable states and a negative expectation value for at least one entangled state. The advantage of entanglement witnesses over other methods such as, for example, full state tomography is that they require less measurements, and thus less experimental effort to detect and prove the existence of entanglement for a given (mixed) state. Witnesses have intensively been used in experiments with photons $[17,18]$ and trapped ions [19], but so far only a few theoretical proposals exist for using witness operators in solid state nanosystems [20].

This paper is organized as follows: in section 2 we briefly summarize the mechanisms influencing the timescales $T_{1}$ and $T_{2}$ for electron spin qubits. We describe two theoretical models of dephasing for these qubits and compare them to the heuristic master equation model. We also show how we calculate the 
decoherence of a multipartite state of separated qubits using the Lindblad formalism. Next (section 3) we demonstrate our main ideas and methods using the simplest case of two qubits, compare the different models of decoherence and introduce our filtered witness operator. In section 4 we consider both GHZ and W states for three qubits. In section 5 we do the same for four qubits and consider in addition the cluster and Dicke entanglement classes. We also discuss the dependence of the decay of entanglement on the initial state. Finally, in section 6, we discuss the case of $N$ qubits: we show that the entanglement of a specific GHZ state can theoretically be detected for any finite time, and discuss the feasibility of generating and detecting many-qubit entangled states of electron spin qubits based on decoherence and operational timescales that have been measured in recent experiments on single and double quantum dots.

\section{Decoherence model}

Decoherence is caused by uncontrolled interactions between the qubit and the environment [21]. This effect is usually characterized by two timescales, the phase randomization time $T_{2}$ ('dephasing time') and the time $T_{1}$ in which the excited state $|1\rangle$ relaxes to the ground state $|0\rangle$ by energy exchange with the environment ('relaxation time') [11]. For electron spin qubits (as for most solid state qubits) the dephasing time is much shorter than the relaxation time, $T_{2} \ll T_{1}$, and is therefore the dominant timescale for the loss of quantum correlations. In this section, we consider both a simple exponential model of decoherence based on these two timescales, as well as use two microscopic descriptions of dephasing for electron spin qubits in quantum dots to derive more sophisticated time evolutions of decoherence.

We start by briefly discussing decoherence mechanisms for electron spin qubits. The original idea by Loss and DiVincenzo [22] proposed to confine single electrons in a quantum dot (an island of charge in a two-dimensional electron gas) and apply a magnetic field to split the degeneracy of the spin-up and spin-down states, thus creating a two-level system serving as a carrier for quantum information: an electron spin qubit. Two electron spin qubits interact via a Heisenberg coupling, and this interaction can be controlled by tuning the potential barrier between two neighboring dots [23]. Single-qubit operations rely on electron spin resonance and can be performed by applying local electric or magnetic fields [24, 25].

Decoherence-interaction with the environment-is mainly mediated by two processes, spin-orbit interaction and hyperfine interaction [8]. Spin-orbit interaction does not have a direct effect on the electron spin, since the electrons do not move, but it leads to a mixing of spin and orbital degrees of freedom [26]. In GaAs quantum dots, spin-orbit interaction is estimated to be small-both experimentally [27] and theoretically [28] — compared to the hyperfine interaction with the nuclei, so that the latter is the dominant source of dephasing.
If the atoms of the semiconductor material have a non-zero nuclear magnetic moment (such as, for example, in GaAs; in other materials, such as purified $\mathrm{SiC}$, this effect is not present), the electron spin $\vec{S}$ interacts with the nuclear spins via the hyperfine interaction [29]: the Hamiltonian for such a system can be written as $[31,32]$

$$
\mathcal{H}_{h f}=b_{0} S_{z}+\epsilon_{n z} I_{z}+\vec{h} \cdot \vec{S}
$$

Here, $b_{0}=g^{*} \mu_{B} B_{0}\left(\epsilon_{n z}=g_{I} \mu_{n} B_{0}\right)$ is the electron (nuclear) Zeeman splitting (calculated using the Bohr (nuclear) magneton $\mu_{\mathrm{B}}\left(\mu_{N}\right.$, where $\left.\mu_{N} \ll \mu_{\mathrm{B}}\right)$ and the effective $g$ factor of the electron (nuclei), $g^{*}\left(g_{I}\right)$, which in GaAs takes the value $\left.g^{*}=-0.44\right)$. Next, $I_{z}=\sum_{k=0}^{n-1} \vec{I}_{z}^{k}$ is the sum over the $z$ component of all nuclear spins $\vec{I}^{k}$, and $\vec{h}=\sum_{k=0}^{n-1} A_{k} \vec{I}_{k}$ denotes the quantum field of the nuclei acting on the electron spin, where $n$ are the number of nuclei whose wavefunction overlaps with the electron's wavefunction $\left(n \approx 10^{5}\right.$ for typical dots), $A_{k}$ denotes the coupling strength between the $k$ th nucleus and the electron. Since the electron's wavefunction is zero outside the dot, there is no overlap with the nuclei outside the quantum dot-thus each electron in the array couples to a different bath of nuclei, and the decoherence is thus local, as stated above. Since hyperfine interaction is the dominant source of noise, we can disregard other types of noise which might induce some correlations between different qubits, such as, for example, phonons.

For an intuitive semiclassical description of decoherence due to hyperfine interaction the quantum field of the nuclear spins can be treated as an additional (classical) magnetic field-the Overhauser field-by replacing $g^{*} \mu_{\mathrm{B}} \vec{B}_{n} \equiv \vec{h}$. The maximum value this field can reach in GaAs is about [30] $B_{\max }=5 \mathrm{~T}$ for fully polarized nuclei. In low external magnetic fields, the Overhauser field undergoes Gaussian fluctuations around a root-mean-square value [31-33] of $B_{\max } / \sqrt{n}$. The electron thus feels a total magnetic field which consists of the sum of the controlled external field $\vec{B}_{0}$ and the random Overhauser field $\vec{B}_{n}$. The field's longitudinal component $B_{\text {nuc }}^{z}$ (parallel to $\vec{B}_{0}$ ) changes the precession frequency of the electron spin by $h_{z}=g^{*} \mu_{\mathrm{B}} B_{\text {nuc }}^{z}$. The transverse part $B_{\text {nuc }}^{x, y}$ changes the precession frequency even only in quadratic order, $\approx g^{*} \mu_{\mathrm{B}}\left(B^{x, z}\right)_{\text {nuc }}^{2} / B_{0}$. This random nuclear magnetic field changes in time: two nuclei with different coupling strength $A_{k}$ can exchange their spin, thus leading to a change in the Overhauser field $B_{n}$; these fluctuations appear on a timescale of 10-100 $\mu$ s (for a weak external field) [36], but could probably be extended up to more than several seconds to minutes (for the longitudinal nuclear field $B_{\text {nuc }}^{z}$ in a strong external field $\left.B_{0}\right)^{5}$.

The bulk dephasing time $T_{2}^{*}$ (at which the fluctuating nuclear magnetic field removes the phase information) can be measured by rotating the spin in the $x y$ plane, let it evolve freely, and then rotate it back along the $z$ axis for measurement (so-called spin echo measurements). Each data point then has to be averaged over many measurements, during which the nuclear field evolves. This leads to an average dephasing time $T_{2}^{*}$, which has been measured to be about $100 \mathrm{~ns}$ [38].

5 This is not confirmed experimentally, but the very slow decay of nuclear spin polarization of up to several minutes is an indication for slow change of $B_{\text {nuc }}^{z}$; see, e.g., [37]. 
The dephasing time $T_{2}$ of a single electron, on the other hand, is very hard to measure, because it is not possible to measure the initial orientation and strength of the nuclear field with sufficient precision. Estimates in various regimes predict $T_{2} \sim 1-100 \mu \mathrm{s}$ : a good way to estimate $T_{2}$ is by using a Hahn echo technique, where the free evolution of the spin due to the initial magnetic field is undone by reversing the spin, but not the dephasing due to the change of this nuclear field [41]. Assuming Gaussian fluctuations of the nuclear field on a timescale of $10 \mathrm{~s}$ and (a conservatively estimated) $T_{2}^{*}=10 \mathrm{~ns}$, a time $T_{2}=10 \mu \mathrm{s}$ can be extracted [8], which has been confirmed by measurements [23] providing a lower bound on $T_{2}$ of $1.2 \mu \mathrm{s}$.

For a microscopic, quantum-mechanical treatment of $\vec{h}$, we rewrite $\mathcal{H}_{h f}$ in (1) in a parallel and transversal part [31, 32, 39, 40]:

$$
\mathcal{H}_{h f}=\underbrace{\left(b_{0}+h_{z}\right) S_{z}}_{\mathcal{H}_{h f}^{0}}+\underbrace{\frac{1}{2}\left(h_{+} S_{-}+h_{-} S_{+}\right)}_{V} .
$$

$V$ describes a flip-flop interaction between the electron and a nucleus, thus the operators are the raising and lowering operators for the $\operatorname{spin}\left(S_{ \pm}=S_{x} \pm \mathrm{i} S_{y}\right)$ and a nucleus $\left(h_{ \pm}=h_{x} \pm \mathrm{i} h_{y}\right)$. This perturbation $V$ is small as soon as there is some external magnetic field and the energy mismatch between the electron and the nuclear spin states suppresses it, as discussed above: expressed in numbers, this requires $|A| \ll 2\left|g^{*} \mu_{B} \vec{B}_{0}-g_{I} \mu_{N} \vec{B}_{0}+2 p I A\right|$ [39] or equivalently (using $\mu_{B} \gg \mu_{N}$ and low polarization) $|A| \ll 2\left|g^{*} \mu_{B} B_{0}\right|$, which is fulfilled in typical experiments (with an external field above $\gtrsim 3 \mathrm{~T}$; in $[35,46]$, for example, fields of up to $8 \mathrm{~T}$ are used). A first approximation is to completely neglect this term and only consider the change of precession frequency due to the nuclear field $h_{z}$. Using the central limit theorem for a large number of nuclear spins results in a Gaussian distribution for $h_{z}$. The transverse correlator, defined as the self-correlation function of the transverse spin component, $\left\langle S_{+}\right\rangle_{t}=\operatorname{Tr}\left[\mathrm{e}^{\mathrm{i} \mathcal{H} h_{h}^{0} t} S_{+} \mathrm{e}^{-\mathrm{i} \mathcal{H}_{h f}^{0} t} \rho(0)\right]$ (here, $\rho(0)$ is the initial density matrix of the combined system of electron and nuclei), is given by $[39,40]$

$$
\left\langle S_{+}^{(\mathrm{se})}\right\rangle_{t}=\left\langle S_{+}\right\rangle_{0} \exp \left[-\frac{t^{2}}{2 \tau_{\mathrm{se}}^{2}}+\frac{\mathrm{i}}{\hbar}\left(b+\left\langle h_{z}\right\rangle\right) t\right] .
$$

As opposed to exponential decay of phase coherence with timescale $T_{2}$, (3) represents superexponential decay with a characteristic time $\tau_{\mathrm{se}} \equiv 2 \hbar / A \sqrt{n /\left(1-p^{2}\right)}$ : for a GaAs dot with almost no polarization, $p \ll 1$, one can estimate $\tau_{\mathrm{se}} \approx 5 \mathrm{~ns}$, which is much faster than the experimentally observed $T_{2}$ time. The second, imaginary part represents the coherent rotation induced by the total magnetic field. The value for $\left\langle h_{z}\right\rangle$ depends on the initial state of the nuclei: for a pure state with each nucleus having probability $(1+p) / 2$ for being in the excited state, it can directly be calculated as $\left\langle h_{z}\right\rangle=p A / 2$, where $A$ is the hyperfine coupling field.

A more sophisticated approach is to include the perturbation term $V$ in (2) and rewrite the von Neumann equation in the form of a Nakajima-Zwanzig generalized master equation (GME) [39]:

$$
P \dot{\rho}(t)=-\mathrm{i} P L P \rho(t)-\mathrm{i} \int_{0}^{t} \Sigma\left(t-t^{\prime}\right) \rho\left(t^{\prime}\right) \mathrm{d} t^{\prime} .
$$

Here $P$ is the projector on the electron subspace, $L$ the Liouville operator $(L \mathcal{O} \equiv[\mathcal{H}, \mathcal{O}]$ for any operator $\mathcal{O})$ and $\Sigma(t)$ the self-energy superoperator. Using regular perturbation theory in the parameter $1 / b_{0}$ (i.e. for a high magnetic field $b_{0} \gg A$ ), some (unphysical) secular terms arise; these terms do not occur by directly expanding $\Sigma(t)$ in the GME. The latter results in a self-correlation function for the transversal spin of the form [39] (in the frame oscillating with a frequency proportional to the Zeeman splitting)

$$
\left\langle S_{+}^{(b m)}\right\rangle_{t}=\left\langle S_{+}\right\rangle_{t}+R_{+}(t) \mathrm{d} t,
$$

where $\left\langle S_{+}\right\rangle_{t}$ is the Markovian solution and $R_{+}(t)$ is the remainder term, i.e. the difference between the exponential and the non-Markovian solution in the Born approximation. This can be written as $R_{+}(t)=\mathrm{i} \int_{0}^{t} \Psi\left(t-t^{\prime}\right)\left\langle S_{+}^{(b m)}\right\rangle_{t^{\prime}} \mathrm{d} t^{\prime}$ and solved by iterating to leading order in the parameter $\delta=$ $A /\left(4 N\left[b_{0}+h_{z}\right]\right)$ (corresponding to a high external field, since $\left.\delta \sim A / b_{0}\right)$. The solution depends strongly on the wavefunction of the electron: we assume the electron to have a Gaussian wavefunction in two dimensions, resulting in

$$
\begin{aligned}
R_{+}(t) & \simeq-\delta\left\langle S_{+}\right\rangle_{0} \exp \left[\frac{\mathrm{i} t A N}{2 \hbar \tau_{\mathrm{bm}}\left(b_{0}+h_{z}\right)}\right]+\frac{\delta \tau_{\mathrm{bm}}^{2}}{t^{2}} \\
& \times\left(-1+\cos \left[\frac{t}{\tau_{\mathrm{bm}}}\right]+p \frac{t}{\tau_{\mathrm{bm}}} \sin \left[\frac{t}{\tau_{\mathrm{bm}}}\right]\right. \\
& \left.+\mathrm{ip}\left\{\frac{t}{\tau_{\mathrm{bm}}} \cos \left[\frac{t}{\tau_{\mathrm{bm}}}\right]-\sin \left[\frac{t}{\tau_{\mathrm{bm}}}\right]\right\}\right) .
\end{aligned}
$$

Here we have defined a characteristic time $\tau_{\mathrm{bm}}=2 n \hbar / A$ ( $\tau_{\mathrm{bm}} \approx 1 \mu \mathrm{s}$ for GaAs quantum dots). In a realistic setting, this correction term $R_{+}(t)$ is very small, since $\delta$ is very small: in GaAs typically $\delta \approx 10^{-6}$. Nonetheless, we will calculate this correction for completeness.

Relaxation of an electron spin qubit is caused by the same two effects as dephasing: spin-orbit and hyperfine interaction. The required energy for the spin-orbit interaction to flip the spin of the electron is provided by the phonons in the lattice of the semiconductors forming the 2DEG, and can be calculated as a function of the external magnetic field $B_{0}$ [26]. The hyperfine contribution to relaxation manifests itself as flips of the electron spin through exchanging its spin state with a nuclear spin. For increasing external field, the energy mismatch between the nuclear spin states and the electron spin state grows, and more and more energy has to be absorbed by phonons - thus the relaxation can be suppressed by applying a higher external field. The relaxation time $T_{1}$ has been measured in experiments to range from $170 \mathrm{~ms}$ (at $B_{0}=1.75 \mathrm{~T}$ ) to $120 \mu \mathrm{s}\left(B_{0}=14 \mathrm{~T}\right)[34,35]$.

In a phenomenological model of decoherence, the timescales $T_{1}$ and $T_{2}$ are incorporated into a master equation model for the density matrix with $T_{1}$ on the diagonal 
(describing the effect of relaxation) and $T_{2}$ on the off-diagonal (describing dephasing):

$$
\frac{\mathrm{d} \varrho}{\mathrm{d} t}=\left[\begin{array}{cc}
\left(1 / T_{1}\right) \varrho_{22} & -\left(1 / T_{2}\right) \varrho_{12} \\
-\left(1 / T_{2}\right) \varrho_{21} & -\left(1 / T_{1}\right) \varrho_{22}
\end{array}\right]
$$

Qualitatively, the off-diagonal phase components decrease exponentially with a rate $1 / T_{2}$, and the ground state $\varrho_{11}$ becomes populated at the expense of the excited state $\varrho_{22}$, where the normalization condition $(\operatorname{Tr}[\rho(t)]=1)$ has to be fulfilled at any time $t$. Equation (7) is a general phenomenological model to describe decoherence, and can thus be adjusted to describe decoherence for a wide range of systems, but it does not include microscopic information about the quantum processes causing the decoherence.

We now discuss how to extend these decoherence models (equations (3), (5) and (7)) to more than one qubit. For the exponential decay, this is quite straightforward: we rewrite (7) in the Lindblad formalism [42] using the Lindblad operator $\mathcal{L}$ :

$$
\begin{aligned}
\mathcal{L} \varrho= & \frac{\Gamma_{1}}{2}\left(2 \sigma_{+} \varrho \sigma_{-}-\sigma_{-} \sigma_{+} \varrho-\varrho \sigma_{-} \sigma_{+}\right) \\
& +\frac{\Gamma_{2}}{2}\left(2 \sigma_{\mathrm{s}} \varrho \sigma_{\mathrm{s}}-\sigma_{\mathrm{s}} \sigma_{\mathrm{s}} \varrho-\varrho \sigma_{\mathrm{s}} \sigma_{\mathrm{s}}\right) .
\end{aligned}
$$

Here, $\sigma_{ \pm}=1 / 2\left(\sigma_{x} \pm \mathrm{i} \sigma_{y}\right)$ and $\sigma_{\mathrm{s}}=\sigma_{-} \sigma_{+}$are products of the Pauli matrices. Comparing the density matrices resulting from equations (7) and (8), we can identify $\Gamma_{1}=1 / T_{1}$ and $\Gamma_{1}+$ $\Gamma_{2}=2 / T_{2}$. The time evolution of a single qubit is then found by solving $\mathcal{L} \varrho(t)=\mathrm{d} \varrho / \mathrm{d} t$. To extend (8) to multipartite states, we write the Lindblad operator for the $k$ th qubit as $\mathcal{L}_{k}=\mathbb{I} \otimes$ $\ldots \otimes \mathbb{I} \otimes \mathcal{L} \otimes \mathbb{I} \otimes \ldots \otimes \mathbb{I}$, where $\mathcal{L}$ is the $k$ th operator of a total of $N$. The time evolution of the total $N$-partite state is then given by solving as before $\mathcal{L}_{N} \varrho(t)=\mathrm{d} \varrho / \mathrm{d} t$, with $\mathcal{L}_{N}=\sum_{k=1}^{N} \mathcal{L}_{k}$. By using this definition we implicitly assumed that the decoherence of each qubit is governed by the same $\Gamma_{1}$ and $\Gamma_{2}$.

For the two other models, given in (3) and (5), we construct the density matrix of the entangled states in a similar manner. Since the decoherence of the various qubits is assumed to be independent, we can just multiply the corresponding single matrix entries. For statistically distributed quantities (such as, for example, $\left\langle h_{z}\right\rangle$ ), we have to consider the addition rules for distributions with the corresponding variances, and furthermore we have to take into account which contributions have to be conjugated (e.g. the precession terms due to the magnetic field).

\section{Two qubits}

Let us first explain our methods and definitions for the simple case of two qubits. A separable state $\varrho_{\mathrm{s}}$ is defined as a state which can be written as a convex combination of product states $[1,2,4]$ :

$$
\varrho_{\mathrm{s}}=\sum_{i} p_{i} \varrho_{i}^{A} \otimes \varrho_{i}^{B},
$$

where $\varrho^{A}$ and $\varrho^{B}$ are states in different subsystems $A$ and $B$ and the probabilities $p_{i}$ have to fulfill the normalization condition $\sum_{i} p_{i}=1$. If a state is not of this form, it is called entangled.
We use witness operators $[6,12-16]$ to investigate the entanglement of various states. An observable $\mathcal{W}$ is called an entanglement witness if it fulfills the following two requirements:

(i) For any separable state $\varrho_{\mathrm{s}}$, the expectation value of $\mathcal{W}$ is larger than zero: $\operatorname{Tr}\left[\mathcal{W} \varrho_{\mathrm{s}}\right] \equiv\langle\mathcal{W}\rangle_{\varrho_{\mathrm{s}}} \geqslant 0$ for all $\varrho_{\mathrm{s}}$ separable.

(ii) There must be at least one entangled state $\varrho_{e}$ for which $\mathcal{W}$ has a negative expectation value: there exists a $\varrho_{e}$ entangled for which $\langle\mathcal{W}\rangle_{\varrho_{e}}<0$.

Therefore, a measured negative expectation value of the witness guarantees that the state is entangled. For the experimental implementation, entanglement witnesses can be decomposed into local measurements (see also below), and they usually require much fewer measurements than procedures such as full state tomography. Thus they are experimentally easier to implement. Finally, it should be noted that witnesses can be used to quantify entanglement, by giving lower bounds on entanglement measures [43].

The witnesses we use in this paper are derived from the so-called projector-like witness [17]:

$$
\mathcal{W}_{\psi}=c \mathbb{I}-|\psi\rangle\langle\psi|
$$

with the constant $c$ standing for the maximum overlap between the state $|\psi\rangle$ and any separable state. Physically, this witness encodes the fact that, if a state $\varrho$ has a fidelity $F=$ $\operatorname{Tr}[\varrho|\psi\rangle\langle\psi|]$ larger than $c$, then $\varrho$ must be entangled.

We first investigate the time evolution of the Bell state $[1,3]\left|\Psi^{-}\right\rangle \equiv \frac{1}{\sqrt{2}}(|01\rangle-|10\rangle)$. The choice of this Bell state, the singlet state, is motivated by the fact that it is the ground state of the quantum system consisting of two electron spins in a double quantum dot [8]. Thus it is the simplest entangled state that can be created in quantum dots.

The density matrix of a singlet state under exponential decay can be found from equations (7) and (8):

$$
\varrho_{\Psi^{-}}(t)=\frac{1}{2}\left[\begin{array}{cccc}
2[1-\alpha(t)] & 0 & 0 & 0 \\
0 & \alpha(t) & -\beta(t) & 0 \\
0 & -\beta(t) & \alpha(t) & 0 \\
0 & 0 & 0 & 0
\end{array}\right],
$$

with the factors $\alpha(t)=\exp \left[-t / T_{1}\right] \equiv \exp \left[-\Gamma_{1} t\right]$ for relaxation and $\beta(t)=\exp \left[-2 t / T_{2}\right] \equiv \exp \left[-\left(\Gamma_{1}+\Gamma_{2}\right) t\right]$ for dephasing. With that, the fidelity [7] $F=$ $\operatorname{Tr}\left[\left|\Psi^{-}\right\rangle\left\langle\Psi^{-}\right| \varrho_{\Psi^{-}}(t)\right]$ is given by

$$
F(t)=\frac{1}{2}[\alpha(t)+\beta(t)],
$$

and the expectation value of the projective witness for $\left|\Psi^{-}\right\rangle$is then calculated using (10):

$$
\operatorname{Tr}\left[\mathcal{W}_{\mathrm{S}} \rho_{\Psi^{-}}(t)\right] \equiv\left\langle\mathcal{W}_{S}\right\rangle_{\Psi^{-}}(t)=\frac{1}{2}[1-\alpha(t)-\beta(t)],
$$

where $\mathcal{W}_{\mathrm{S}}$ is the witness for the singlet state, $\mathcal{W}_{\mathrm{S}} \equiv \mathbb{I} / 2-$ $\left|\Psi^{-}\right\rangle\left\langle\Psi^{-}\right|$. Figure 1 shows the decay of entanglement for this exponential model of decay of the coherence.

For the other two models of decoherence (equations (3) and (5)), we first have to construct the corresponding density 


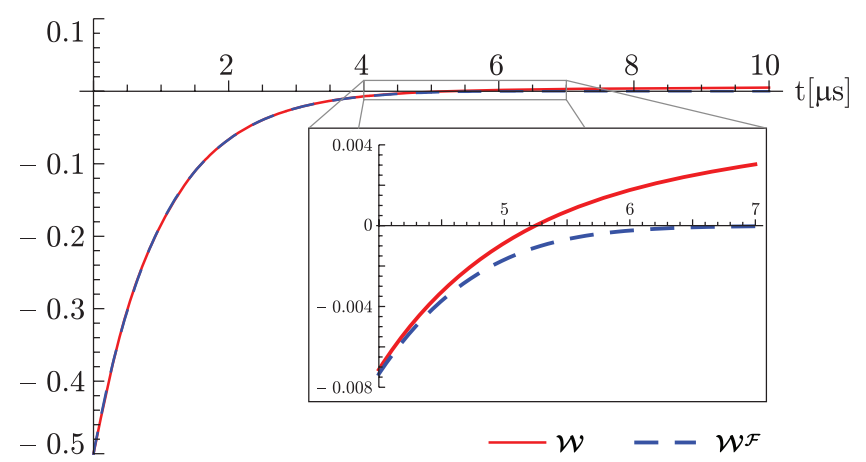

Figure 1. Expectation values for the regular ((13), solid red line) and the filtered ((20), dashed blue line) witness for $\Gamma_{1}=10^{3} \mathrm{~s}^{-1}$ and $\Gamma_{2}=10^{6} \mathrm{~s}^{-1}$. The inset shows a zoom into the region where the regular witness becomes positive. $y$ is chosen such that the witness expectation value becomes minimized at any given time, thus it is time-dependent.

matrix: for the relaxation, we keep the exponential terms $\mathrm{e}^{-t / T_{1}}$, but for the dephasing we use the correlators presented in the previous section. The first model is based on the superexponential dephasing from (3) for each qubit, whose density matrix we label with $\rho_{\mathrm{se}}(t)$. We have to calculate the entries $|01\rangle$ and $|10\rangle$; therefore the conjugation reverses the phase in (3), so the off-diagonal dephasing terms $\left[\rho_{s e}\right]_{(2,3)}=$ $\left[\rho_{s e}\right]_{(3,2)}^{*}$ (the star* stands for complex conjugate) are given in terms of the correlators $\left\langle S_{+}^{(i)}\right\rangle_{t}$ of the $i$ th $\operatorname{dot}(i \in\{1,2\})$ by

$$
\begin{aligned}
{\left[\rho_{s e}\right]_{(2,3)}(t) } & =\left\langle S_{+}^{(1)}\right\rangle_{t}\left\langle S_{+}^{(1)}\right\rangle_{t}^{*} \\
& =\exp \left[-\frac{\tau_{1}^{2}+\tau_{2}^{2}}{2 \tau_{1}^{2} \tau_{2}^{2}} t^{2}+\frac{\mathrm{i}}{\hbar}\left(\left\langle h_{z}^{(1)}\right\rangle-\left\langle h_{z}^{(2)}\right\rangle\right) t\right] \\
& =\exp \left[-\frac{t^{2}}{\tau_{\mathrm{se}}^{2}}\right]
\end{aligned}
$$

where the second line is for identical statistics of the dots (thus with identical characteristic times $\tau_{1}=\tau_{2} \equiv \tau_{\mathrm{se}}$ ). Including the boundary condition $\left[\rho_{s e}\right]_{(2,3)}(0)=-1 / 2$ we obtain the density matrix:

$$
\rho_{s e}(t)=\frac{1}{2}\left[\begin{array}{cccc}
2[1-\alpha(t)] & 0 & 0 & 0 \\
0 & \alpha(t) & -\beta_{s e}(t) & 0 \\
0 & -\beta_{s e}^{*}(t) & \alpha(t) & 0 \\
0 & 0 & 0 & 0
\end{array}\right],
$$

with $\alpha(t)=\exp \left[-t / T_{1}\right]$ as before and $\beta_{s e}(t)=\left[\rho_{s e}\right]_{(2,3)}(t)$ from (14). The witness operator $W_{s e}$ for detecting (15) is thus the same as in (13) with the replacement $\beta(t) \rightarrow \beta_{s e}$. The evolution of the corresponding witness is shown in figure 2(a).

The third model uses the non-Markovian Born approximation for the decay, equations (5) and (6). The singleelectron decay is given by $\left\langle S_{+}^{(\mathrm{bm})}\right\rangle_{t}=\exp \left[-t /\left(2 T_{1}+T_{2}\right)\right]+$ $R_{+}(t)$, where the first exponential term stems from the Markovian solution, and the remainder term is given in (6). In order to set up the density matrix $\rho_{\mathrm{bm}}(t)$ in the nonMarkovian approximation, we replace $\beta(t)$ in (11) by $\beta_{\mathrm{bm}}(t)=$ $\left\langle S_{+}^{(\mathrm{bm})}\right\rangle_{t}\left\langle S_{+}^{(\mathrm{bm})}\right\rangle_{t}^{*}$, in the same way as in the superexponential case.

In the next part we introduce a systematic method to enlarge the time interval during which entanglement can be


Figure 2. (a) Decay of the entanglement witnesses (without (solid) and with (dashed) filter) for the superexponential decoherence model using $\tau_{\mathrm{se}}=4.4 \mathrm{~ns}$ (corresponding to $p=0.1$ ). The inset shows the advantage of the filter operator, which works here as well. (b) Decay of the entanglement witnesses for the non-Markovian approximation, including the correction term to the Markovian solution, for characteristic time $\tau_{\mathrm{bm}}=1 \mu \mathrm{s}$, but an enhanced smallness parameter $\delta=0.1$ in order to underline the effect of the correction term.

detected by the witness operators. This method is based on applying local (so-called filtering) operators to the witness operator and analyzing the measurement results in a different way without requiring more measurements. Analyzing the witness (13), we see immediately that it becomes positive (and hence does not detect the entanglement anymore) when, after some finite time, $\beta(t)$ becomes smaller than $1-\alpha(t)$, though it can be shown (by virtue of the PPT criterion [44], for example) that the state $\varrho_{\Psi^{-}}(t)$ is entangled for any $t<\infty$, i.e. for any $\beta(t)>0$.

Therefore, our goal is to construct a witness operator which is able to detect the entanglement in the state $\varrho_{\Psi^{-}}(t)$ at any time. This can be achieved by a filter operator $\mathcal{F}$ :

$$
\mathcal{F}=\mathcal{F}_{1} \otimes \mathcal{F}_{2}
$$

where the $\mathcal{F}_{i}$ are arbitrary invertible matrices acting on individual qubits. Since $\mathcal{F}$ is local, application of such a filter operator on a state $\rho$ does not change its entanglement properties, i.e. $\mathcal{F}^{\dagger} \varrho \mathcal{F}$ is entangled, iff $\varrho$ is entangled.

Equivalently, one can apply filter operators to witness operators $\mathcal{W}$, and the resulting filtered witness operator $\mathcal{W}^{\mathcal{F}}$ is then given by

$$
\mathcal{W}^{\mathcal{F}}=\mathcal{F} \mathcal{W} \mathcal{F}^{\dagger} .
$$


As normalization, we choose $\operatorname{Tr}[\mathcal{W}]=\operatorname{Tr}\left[\mathcal{W}^{\mathcal{F}}\right]$, to make the witnesses' mean values comparable.

Our goal is now to design a filter $\mathcal{F}_{i}$ such that it increases the negativity of the witness, i.e. it should increase the weight of the terms $\alpha$ and $\beta$ in (13), so that the filtered witness can be used to detect entanglement during longer times. This can be achieved by the following filter:

$$
\mathcal{F}_{i}=\left[\begin{array}{ll}
1 & 0 \\
0 & y
\end{array}\right],
$$

with $y$ a positive real number. The normalized filtered witness for the singlet state then takes the form

$$
\begin{aligned}
\mathcal{W}_{\mathrm{S}}^{\mathcal{F}} & =\left(\mathcal{F}_{1} \otimes \mathcal{F}_{2}\right) \mathcal{W}_{\mathrm{S}}\left(\mathcal{F}_{1} \otimes \mathcal{F}_{2}\right)^{\dagger} \\
& =\frac{1}{1+y^{4}}\left[\begin{array}{cccc}
1 & 0 & 0 & 0 \\
0 & 0 & y^{2} & 0 \\
0 & y^{2} & 0 & 0 \\
0 & 0 & 0 & y^{4}
\end{array}\right],
\end{aligned}
$$

and the expectation value is given by

$$
\left\langle\mathcal{W}_{\mathrm{S}}^{\mathcal{F}}\right\rangle_{\Psi^{-}(t)}=\frac{1}{1+y^{4}}\left[1-\alpha(t)-y^{2} \beta(t)\right]
$$

Clearly, $\left\langle\mathcal{W}_{\mathrm{S}}^{\mathcal{F}}\right\rangle_{\Psi^{-}(t)}$ is negative if $y$ is chosen large enough and time-dependent and $t<\infty$ (thus $\beta(t)>0$ ), and the negativity of the witness can be optimized by a suitable choice of $y \equiv y(t)$ for a given time $t$. The remaining entanglement (which is not detected by $\mathcal{W}_{\mathrm{S}},(13)$ ) in the decohering state can then be detected by measuring this filtered witness operator. The effectiveness of the filter operator crucially depends on the choice of the singlet state $\left|\Psi^{-}\right\rangle$as the initial state: it can easily be shown that, for the other Bell states $\left|\Phi^{ \pm}\right\rangle=\frac{1}{\sqrt{2}}(|00\rangle \pm|11\rangle)$, the filtered witness does not lead to any improvement over the regular witness operator. The decay of entanglement in our model thus strongly depends on the initial state, even within the same basis.

For the experimental implementation, the witness $\mathcal{W}_{\mathrm{S}}^{\mathcal{F}}$ can be decomposed into single-qubit measurements [15]:

$$
\begin{aligned}
\mathcal{W}_{\mathrm{S}}^{\mathcal{F}} & =\frac{1}{4\left(1+y^{4}\right)}\left[\left(1+y^{4}\right)\left(\mathbb{I} \otimes \mathbb{I}+\sigma_{z} \otimes \sigma_{z}\right)-\left(1-y^{4}\right)\right. \\
& \left.\times\left(\sigma_{z} \otimes \mathbb{I}+\mathbb{I} \otimes \sigma_{z}\right)+2 y^{2}\left(\sigma_{x} \otimes \sigma_{x}+\sigma_{y} \otimes \sigma_{y}\right)\right] .
\end{aligned}
$$

This decomposition requires three measurement settings (namely $\sigma_{i} \otimes \sigma_{i}$ with $i \in\{x, y, z\}$ ) instead of the nine settings full state tomography would require $[14,45]$. Similar decompositions exist for all other witnesses occurring in this paper $[17,47]$.

In figures 1 and 2, the evolution of the expectation values for both the regular witnesses (solid line) and the filtered witnesses (dashed line) are plotted. In the experimentally relevant limit $\left(\Gamma_{2} \gg \Gamma_{1}\right)$, the advantage of the filter operator in an experiment does not manifest itself as strongly as would be the case for $\Gamma_{2} \simeq \Gamma_{1}$; however, the principal advantage that the entanglement can be detected for any finite time is demonstrated in the insets by a zoom into the region where the unfiltered witness becomes positive. The filtered witness remains negative, albeit with a small, exponentially decaying absolute value, for any finite time: this proves that the $\left|\Psi^{-}\right\rangle$

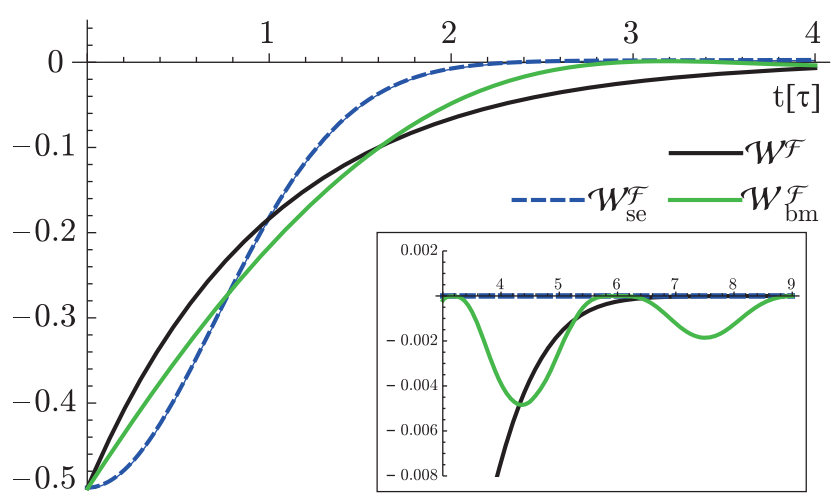

Figure 3. Comparison of the evolution of the filtered witness operators for exponential decay, superexponential decay and the Born approximation, using $\delta=0.1$. The unit of time $\tau$ is the critical time in each model (thus $\tau=T_{2}, \tau_{\mathrm{se}}, \tau_{\mathrm{bm}}$ ). For short times, both the superexponential and the non-Markovian approximation are decaying less strongly than the exponential model, but after some time, the witness assuming exponential decay has a greater negativity. The inset compares the longer-time behavior of the three models: the non-Markovian approximation features a periodic recurrence of negative values (due to precession around the nuclear magnetic field) and the slowest long-time decay (disregarding the coherent precession).

contains at least a very small amount of entanglement at any time under our decoherence models; however, it will not lead to a significant advantage in an experiment, since the noise due to imperfect state preparation and measurement fidelities will render it virtually impossible to measure the expectation value with such a high precision.

From this curve, one can conclude that it becomes difficult to detect entanglement after more than a few $\mu$ s under realistic conditions assuming any of the decay models we have considered (for the superexponential decay even after a few ns, but for all models the exact time also depends on the size of the error bars in a given experiment). Consequently, any generation scheme for the Bell states which requires a generation time longer than this time will probably not work in practice.

Let us conclude this section by a comparison of the three models. The evolution of the expectation values of the filtered witnesses is plotted in figure 3 (all in units of the respective critical time for the comparison). In the nonMarkovian approximation, we have chosen the smallness parameter $\delta=0.1$ such that the effect of the correction can be shown. This comparison reveals benefits and drawbacks of each decay model: first, the filtered entanglement operators are all negative for any finite time $t<\infty$. The entanglement in the superexponential model decays faster than in the other two, and in the non-Markovian approximation we see the effect of the power-law tail (the second term in (6)) as a periodic rebouncing due to the precession around the $z$ component of the nuclear magnetic field, $h_{z}$. As a result, the expectation value of this witness operator is more negative than for the purely exponential decay. In the main plot showing the short time evolution scaled by the critical time for each model, the differences between the models are not as pronounced, and they behave roughly the same. 
Let us consider a realistic experiment at this point: in an experiment, it is likely that errors will arise due to an imperfect read-out of the electron spin states [35, 46], which will manifest themselves as error bars on the curves for the time evolution. This error will make it unlikely to detect the entanglement at longer times in a realistic experiment. Regarding figure 3 with these errors in mind, the evolution within the three models is roughly equivalent. Another source of possible experimental inaccuracies is the preparation of the initial state-in general, it will not be the exact aimed-for state, but a mixture of states. This mixture will influence the use of the witness and the filter operator, which as well strongly depends on the nature of the mixture; though the precise influence is hard to predict, the filter will always improve the witness operator to some degree. However, the creation of pure singlet states in double quantum dots has already been experimentally achieved in a controllable manner and with a high probability of success [23]. Therefore we expect that our noiseless results can nevertheless be used to give qualitative predictions of the decay of entanglement.

So far, we have considered two entangled qubits and found that their entanglement remains detectable for about the same time for three decoherence models. When considering generalization to many qubits, we note that the exponential model features a large advantage compared to the other two, since for this model there is a general method for calculating the time evolution of the density matrix for an arbitrary number of qubits (see also section 6), whereas for the two other models we have to construct the density matrix for each new state by hand. Therefore, in the following sections, we will use the exponential model for the generalization to multiple qubits.

\section{Three qubits}

For three or more particles, the situation is more complicated, since different classes of multiparticle entanglement exist [48-50].

Let us first discuss the notion of partial separability. A state can be partially separable, meaning that some of the qubit states are separable, but not all. An example for three particles is the state

$$
\left|\psi^{b s}\right\rangle=\left|\phi^{A B}\right\rangle \otimes\left|\phi^{C}\right\rangle,
$$

where $\left|\phi^{A B}\right\rangle$ is a (possibly entangled) state of two qubits (defined on subsystems $A$ and $B$ ) and $\left|\phi^{C}\right\rangle$ a state of the third qubit (defined on subsystem $C$ ). The state $\left|\psi^{b s}\right\rangle$ is separable with respect to a certain bipartite split, so it is called biseparable. A mixed state is biseparable if it can be written as a mixture of biseparable pure states.

If a state is not biseparable, it is genuinely multipartite entangled. There exist different classes of multipartite entangled states [48] and the number of entanglement classes increases with the number of qubits [51]. An entanglement class can be defined by the following question: given a single copy of two pure states $|\psi\rangle$ and $|\phi\rangle$, is it possible, at least in principle, to transform $|\psi\rangle$ into $|\phi\rangle$ (and vice versa) using local transformations only? Even if the probability of success is small? For three qubits, for example, two entanglement classes exist, the GHZ and the W class. Every genuine multipartite entangled three-qubit state can be transformed into one of the two states [48]:

$$
\begin{gathered}
\left|\mathrm{GHZ}_{3}\right\rangle=\frac{1}{\sqrt{2}}(|010\rangle+|101\rangle), \\
\left|W_{3}\right\rangle=\frac{1}{\sqrt{3}}(|100\rangle+|010\rangle+|001\rangle),
\end{gathered}
$$

but, remarkably, these two states cannot (not even stochastically) be transformed into each other and are therefore representatives of different entanglement classes.

Let us now investigate the lifetime of these two states using our exponential decoherence model, described below (8). After calculating the time evolution of the two states, we obtain for the corresponding fidelities:

$$
\begin{aligned}
& F_{\mathrm{GHZ}}(t)=\frac{1}{4}\left(\exp \left[-2 \Gamma_{1} t\right]+\exp \left[-\Gamma_{1} t\right]\right. \\
& \left.\quad+2 \exp \left[-\frac{3}{2}\left(\Gamma_{1}+\Gamma_{2}\right) t\right]\right), \\
& F_{W}(t)=\frac{1}{3}\left(\exp \left[-\Gamma_{1} t\right]+2 \exp \left[-\left(\Gamma_{1}+\Gamma_{2}\right) t\right]\right) .
\end{aligned}
$$

From these fidelities, the expectation values of the witnesses can directly be determined as $\left\langle\mathcal{W}_{G}\right\rangle_{\rho_{G}(t)} \equiv 1 / 2-F_{\mathrm{GHZ}}(t)$ for the GHZ state and $\left\langle\mathcal{W}_{W}\right\rangle_{\rho_{W}(t)} \equiv 2 / 3-F_{W}(t)$ for the W state.

Our next step is to apply the filter operators to the witnesses. This yields the following values for the expectation value of the filtered witness operators:

$$
\begin{aligned}
& \left\langle\mathcal{W}_{G}^{\mathcal{F}}\right\rangle_{\varrho_{G}(t)}=\frac{3}{2\left(1+2 y^{2}+2 y^{4}+y^{6}\right)}\left[2+\left(-3+2 y^{2}\right) \mathrm{e}^{-\Gamma_{1} t}\right. \\
& \left.\quad+\left(1-2 y^{2}\right) \mathrm{e}^{-2 \Gamma_{1} t}-2 y^{3} \mathrm{e}^{-\frac{3}{2}\left(\Gamma_{1}+\Gamma_{2}\right) t}\right] \\
& \left\langle\mathcal{W}_{W}^{\mathcal{F}}\right\rangle_{\varrho_{W}(t)}=\frac{13}{3\left(2+3 y^{2}+6 y^{4}+2 y^{6}\right)} \\
& {\left[2+\left(-2+y^{2}\right) \mathrm{e}^{-\Gamma_{1} t}-2 y^{2} \mathrm{e}^{-\left(\Gamma_{1}+\Gamma_{2}\right) t}\right]}
\end{aligned}
$$

These witness operators can be measured by four (for the GHZ state) or five (for the W state) measurement settings [47], compared to the 27 measurement settings required for full state tomography. In principle, the witness for the W state can be improved by taking the projector onto the subspace with at most two excitations [16] $\left(\mathbb{I}_{2}=\mathbb{I}-|111\rangle\langle 111|\right.$ instead of $\mathbb{I}$ ). However, in the present case this does not give any improvement, since the $|111\rangle\langle 111|$ state is not populated. The time evolution of the witness expectation values (27) and (28) are plotted in figure 4 (for $\Gamma_{1}=10^{3} \mathrm{~s}^{-1}$ and $\Gamma_{2}=10^{6} \mathrm{~s}^{-1}$ ).

\section{Four qubits}

The more qubits that are added, the more distinct classes of entangled states arise. For four qubits, we investigate the following four classes:

$$
\begin{aligned}
& \left|\mathrm{GHZ}_{4}\right\rangle=\frac{1}{\sqrt{2}}(|0101\rangle+|1010\rangle) \\
& \left|C_{4}\right\rangle=\frac{1}{2}(|0101\rangle+|0110\rangle+|1001\rangle-|1010\rangle) \\
& \left|W_{4}\right\rangle=\frac{1}{2}(|1000\rangle+|0100\rangle+|0010\rangle+|0001\rangle), \\
& \left|D_{4}\right\rangle=\frac{1}{\sqrt{6}}(|0011\rangle+|0101\rangle+|0110\rangle \\
& \quad+|1001\rangle+|1010\rangle+|1100\rangle)
\end{aligned}
$$




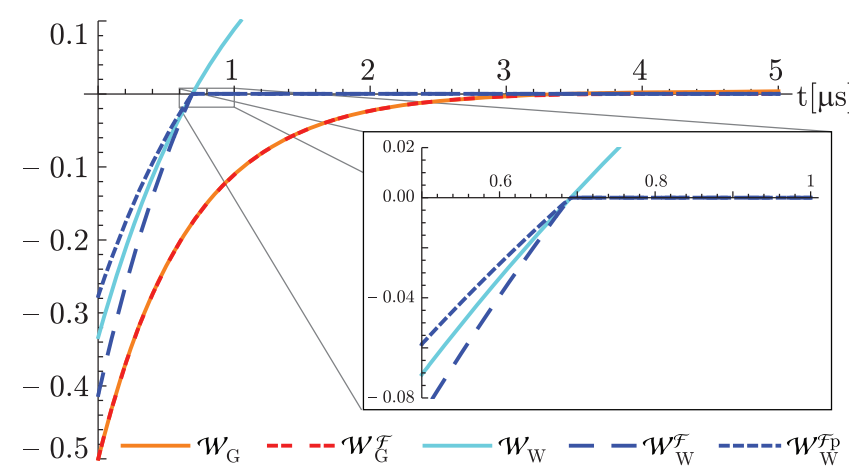

Figure 4. Expectation values for the witnesses for the tripartite GHZ and W states, both regular (solid lines) and filtered (dashed), as a function of time $t$. The projected filter $\mathcal{W}_{W}^{\mathcal{F} p}$ (see text) is plotted as well, though it is not better than the filtered witness $\mathcal{W}_{W}^{\mathcal{F}}$. Parameters used are $\Gamma_{1}=10^{3} \mathrm{~s}^{-1}$ and $\Gamma_{2}=10^{6} \mathrm{~s}^{-1}$. As in figure 1, $y$ is time-dependent and chosen so as to minimize the witness expectation value.

All of these states have been realized in various experiments for different physical systems ${ }^{6}$, but so far not in solid state nanosystems. Also, some of their decoherence properties have been investigated from different theoretical perspectives [53, 54]. The states $\left|\mathrm{GHZ}_{4}\right\rangle$ and $\left|W_{4}\right\rangle$ are the fourqubit versions of the states we have investigated for three qubits in the previous section. $\left|C_{4}\right\rangle$ is a representative of the so-called cluster class [55], important in the context of one-way quantum computing [56]. The Dicke state [57] $\left|D_{4}\right\rangle$ is an extension of the $\mathrm{W}$ state and consists of all possible permutations of states containing two excitations. The fidelity of these states evolves as

$$
\begin{aligned}
& F_{\mathrm{GHZ}_{4}}(t)=\frac{1}{2}\left(\exp \left[-2 \Gamma_{1} t\right]+\exp \left[-2\left(\Gamma_{1}+\Gamma_{2}\right) t\right]\right), \\
& F_{C_{4}}(t)=\frac{1}{2}\left(\exp \left[-2 \Gamma_{1} t\right]+\exp \left[-2\left(\Gamma_{1}+\Gamma_{2}\right) t\right]\right. \\
& \left.\quad+\exp \left[-\left(2 \Gamma_{1}+\Gamma_{2}\right) t\right]\right), \\
& F_{W_{4}}(t)=\frac{1}{4}\left(\exp \left[-\Gamma_{1} t\right]+3 \exp \left[-\left(\Gamma_{1}+\Gamma_{2}\right) t\right]\right), \\
& F_{D_{4}}(t)=\frac{1}{6}\left(\exp \left[-2 \Gamma_{1} t\right]+\exp \left[-2\left(\Gamma_{1}+\Gamma_{2}\right) t\right]\right. \\
& \left.\quad+4 \exp \left[-\left(2 \Gamma_{1}+\Gamma_{2}\right) t\right]\right) .
\end{aligned}
$$

The corresponding projective witnesses can be found, as before, using $c \mathbb{I}-F(t)$, with $c=1 / 2$ for the cluster and GHZ states, $c=3 / 4$ for the $\mathrm{W}$ state and $c=2 / 3$ for the Dicke state [58].

Again, filter operations can be applied: the resulting formulae are lengthy and therefore not given here. The improvement over the regular witness again shows (as for the general case of $N$ qubits) that the GHZ state contains in theory entanglement for any finite time- but so little that this result is of a theoretical nature and not experimentally relevant. For the other classes of states, the filter can lead to a slightly higher negativity, but not to an extension of the time where the expectation value will become positive. So what is left is to compare the differences in the evolution of the expectation values of these witness operators for the four classes, and to

\footnotetext{
6 See, e.g., [17, 18] and Kiesel et al [19] for realizations of GHZ, W and cluster states of up to eight qubits using photons and ions, respectively, and [52] for a four-qubit Dicke photon state.
}

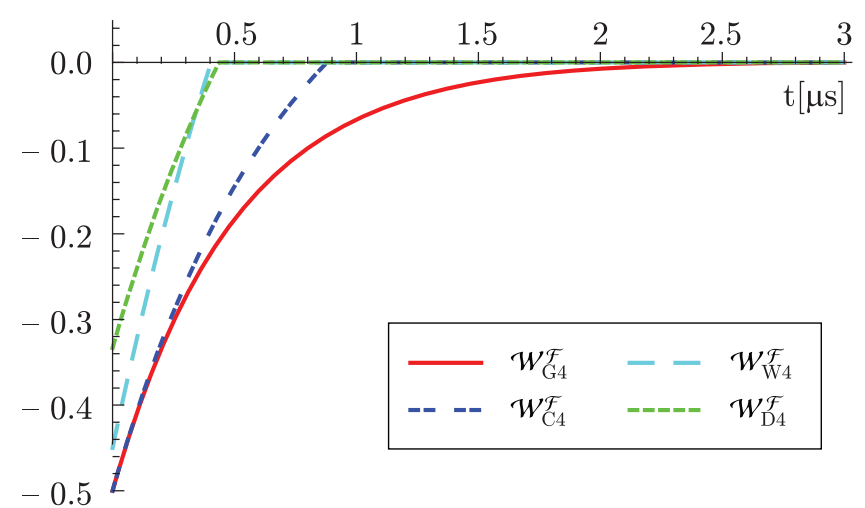

Figure 5. Expectation values for the filtered witness operators for the four considered classes of four-partite entangled states (GHZ, cluster, $\mathrm{W}$ and Dicke). The filter operator makes the entanglement in the GHZ state detectable for arbitrarily long times (at least in principle). The entanglement of the other three classes decays faster, but the cluster state is more stable (decays slower) than both the $\mathrm{W}$ and the Dicke states. Parameters used are the same as in figure 4.

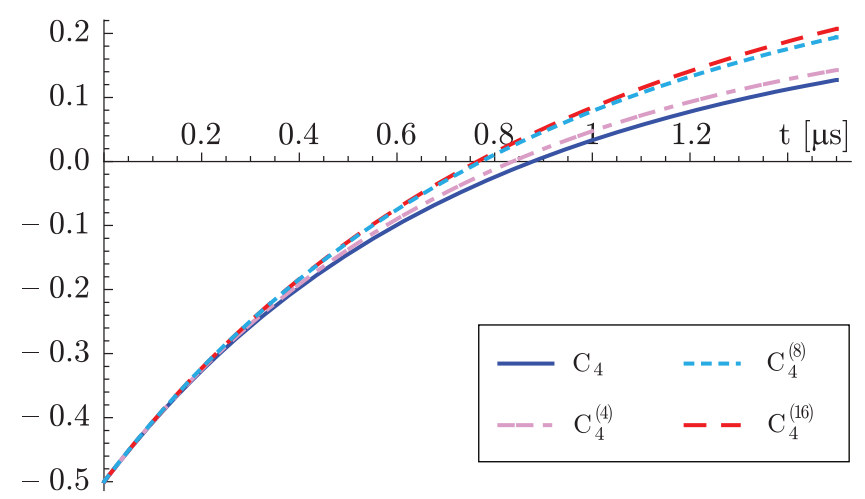

Figure 6. Expectation values of the witnesses for some four-partite cluster states. The upper index indicates the number of terms in the representation of each state, while the state without an index is the one given in (30). Parameters used are the same as in figure 4.

see which one is the most stable, i.e. detectable for the longest time. This is done in figure 5 .

At this point the same question can be asked as for the two-qubit state that we investigated in section 3: how does the available detection time depend on the exact state chosen as representative of a class? Or, equivalently, the fidelity of which state decoheres most slowly? In fact, writing the states above in a different basis leads to different decay rates.

This is illustrated in figure 6 , where the evolution of the witness expectation value of four different cluster states is plotted: $\left|C_{4}\right\rangle$ from (30), $\left|C_{4}^{(16)}\right\rangle$ is the original cluster state from [59] containing 16 terms, and the two additional representations

$$
\begin{aligned}
\left|C_{4}^{(4)}\right\rangle & =\frac{1}{2}(|0000\rangle+|0011\rangle+|1110\rangle+|1101\rangle) \\
\left|C_{4}^{(8)}\right\rangle & =\frac{1}{2^{3 / 2}}(|0000\rangle+|0011\rangle+|0100\rangle+|0111\rangle \\
- & |1000\rangle-|1010\rangle+|1101\rangle+|1110\rangle)
\end{aligned}
$$

with four and eight terms, respectively (the first one is a representation with the minimal number of terms, which will 
be used again in the next section, the second one a rotated version of the original cluster state). As can be seen in figure 6 , the detection time decreases as the number of terms increases, though the effect is not very large for four or more terms. The representations with the minimal number of terms thus decohere more slowly. This is not surprising, since one can prove for a similar decoherence model that states with the minimal number of terms are most robust [53]. For representations with the same number of terms, the number of excitations in each term can influence the detectability: which one of the two is easier to detect then depends on the ratio of $\Gamma_{1}$ and $\Gamma_{2}$.

\section{6. $N$ qubits}

Let us now consider the general situation of $N$ qubits. We concentrate on three types of entangled states for which there exist proposals how to generate them using available singleand two-qubit operations in quantum dots [60]: GHZ, W and cluster states. Our goal is to calculate the time evolution of the normal (unfiltered) witness for arbitrary $N$ and compare this with the time necessary to generate and measure the state. The representatives of the first two classes can be written down straightforwardly (for even $N$ ):

$$
\begin{gathered}
\left|\mathrm{GHZ}_{N}\right\rangle=\frac{1}{\sqrt{2}}(|01 \ldots 01\rangle+|10 \ldots 10\rangle) \\
\left|W_{N}\right\rangle=\frac{1}{\sqrt{N}}(|00 \ldots 01\rangle+|00 \ldots 10\rangle+\cdots+|10 \ldots 00\rangle) .
\end{gathered}
$$

Calculating the expectation value of the witness operators leads to (for even $N$ )

$$
\begin{aligned}
&\left\langle\mathcal{W}_{G}\right\rangle_{\varrho_{G}(t)}=\frac{1}{2}\left\{1-\exp \left[-\frac{N}{2} \Gamma_{1} t\right]\right. \\
&-\exp {\left.\left[-\frac{N}{2}\left(\Gamma_{1}+\Gamma_{2}\right) t\right]\right\}, } \\
&\left\langle\mathcal{W}_{W}\right\rangle_{\varrho_{W}(t)}=\frac{1}{N}\left\{N-1-\exp \left[-\Gamma_{1} t\right]\right. \\
&\left.-(N-1) \exp \left[-\left(\Gamma_{1}+\Gamma_{2}\right) t\right]\right\}
\end{aligned}
$$

The general form of the cluster state- the one we consider here containing the minimal number of terms, namely $2^{N / 2}$-is more complicated; it can be written as ${ }^{7}$

$$
\left|C_{N}\right\rangle=\bigotimes_{k=1}^{n} \frac{\left[|00\rangle+|11\rangle\left(\sigma_{x} \otimes \mathbb{I}\right)\right]}{\sqrt{2}}
$$

where this formula should be understood as an iteration, with the operator $\left(\sigma_{x} \otimes \mathbb{I}\right)$ acting on the Bell state of the next two qubits. For four qubits, this results exactly in the representation $\left|C_{4}^{(4)}\right\rangle$ from (37), the evolution of which is plotted in figure 6.

\footnotetext{
7 In general, a cluster state is defined as an eigenstate of certain local observables [55, 56]; here we choose them as $\mathcal{S}_{1}=\sigma_{z} \sigma_{z} \mathbb{I I} \ldots ; \mathcal{S}_{2}=$ $\sigma_{x} \sigma_{x} \sigma_{x} \mathbb{I} \ldots ; \mathcal{S}_{3}=\mathbb{I} \sigma_{z} \sigma_{z} \sigma_{z} \mathbb{I} \ldots ; \ldots ; \mathcal{S}_{N}=\mathbb{I} \ldots \mathbb{I} \sigma_{x} \sigma_{x}$. This is locally equivalent to the definition as in $[55,56]$; however, the state given in (43) contains the minimal number of terms; see [53].
}

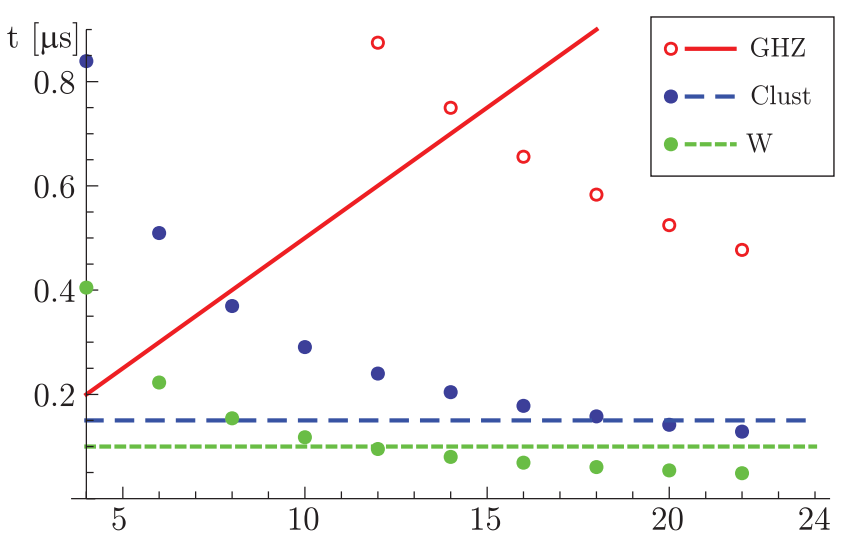

Figure 7. The dots show the time at which the expectation value of the (unfiltered) witness for a given class of states becomes positive as a function of the qubit number $N$. Parameters used are $\Gamma_{1}=10^{3} \mathrm{~s}^{-1}$ and $\Gamma_{2}=10^{6} \mathrm{~s}^{-1}$, except for the cluster state where the relaxation is disregarded $\left(\Gamma_{1}=0\right)$. Also plotted are (shown by lines) the times required to generate the given entangled states, see the text for explanation.

To calculate the time evolution of the fidelity, we represent the cluster state (43) as [56]

$$
\left|C_{N}\right\rangle\left\langle C_{N}\right|=\prod_{k=1}^{N} \frac{\mathbb{I}+S_{k}}{2}
$$

with $S_{k}$ a product of Pauli matrices. We incorporate the effects of dephasing (disregarding the relaxation of the qubits, i.e. setting $\Gamma_{1}=0$, which leads to an error of less than $0.01 \%$ o for four qubits) in every term in the sum of the expanded (44). The resulting fidelity of the cluster state can then easily be calculated numerically up to $N=24$ qubits.

Figure 7 shows the time at which the expectation value of the (unfiltered) projective witness for each of the three states (equations (41), (42) and (44)) becomes positive as a function of the qubit number $N$, as well as a rough estimate of the time necessary to generate and measure these states. For electron spin qubits in quantum dots the generation times are taken from [60]: for both the cluster and the W states the time required to produce these states is independent of the number of qubits, whereas the production time of states of the GHZ class scales linearly with the number of qubits. The measurement times are composed as follows: measurement distinguishes between spin-up and spin-down (defined along the $z$ axis [35]) and measuring the components $\sigma_{x}$ and $\sigma_{y}$ then requires a rotation of the spins by $\pi / 2$, which takes about $\sim 50 \mathrm{~ns}$ [24]. The sum of the generation and the measurement time is given by the lines in the plot.

We see in figure 7 that (as in figures 4 and 5) the entanglement of the GHZ state can be detected for the longest times, but it is more time-consuming to generate than the other two entangled states. Based on the estimates in figure 7, generation and detection of GHZ states should be possible for up to 14 qubits (with the standard projective witness and assuming current operation and decoherence times for electron spin qubits). The cluster state is the state which can be detected for the largest number of qubits, although for up to 
12 qubits the 'time reserve' (i.e. the difference between the time needed for generation and measurement and the time when the expectation value of the witness operator becomes positive) for the GHZ state is somewhat larger than for the cluster state. The W state is the least suitable; the largest state would contain about $\sim 10$ qubits.

Our results for the cluster state show that one-way quantum computing [55] is not really feasible in quantum dots with current dephasing times: we expect that up to maximally $\sim 12$ qubits could be entangled under the presented preparation scheme, which is far too few for exploiting the advantages of a quantum computer.

Based on our assumptions, thus, the simplest state to generate and prove its entanglement would be the GHZ state for up to twelve qubits, and the cluster state for more than twelve and up to twenty qubits, though the remaining entanglement becomes very small. The same holds for the filtered witness for the GHZ state for an arbitrary number of qubits.

\section{Conclusion}

In conclusion, we have investigated entanglement and its detectability in a linear array of electron spin qubits which locally undergo decoherence. We have considered three different phenomenological models for the dephasing of the qubits based on exponential and superexponential decay. Using witness operators as detectors of entanglement and introducing a specific class of filtered witness operators, we estimated the maximum available detection time for entanglement of two electrons using each of the models and found that the time during which entanglement is detectable is independent of the model chosen. We then expanded the exponential model to the case of multipartite entanglement. For three and four qubits, we compared the decay of entanglement for different classes of entangled states with each other, namely the GHZ, W cluster and Dicke classes. We also gave limits on the maximum number of entangled qubits that can be created and measured based on currently known decoherence times for electron spin qubits. The most suitable entangled state turns out to be the GHZ state for up to a few qubits. Our results can help to make a choice as to which state to prepare in experiments. Since local decoherence is characteristic for many types of solid state qubits, our model and the filtered operator technique are applicable to a variety of these qubits.

\section{Acknowledgments}

This work has been supported by The Netherlands Organisation for Scientific Research (NWO), the FWF (START prize) and the EU (SCALA, OLAQUI, QICS).

\section{References}

[1] Einstein A, Podolski B and Rosen N 1935 Phys. Rev. 47777

[2] Schrödinger E 1935 Naturwissenschaften 23807

[3] Bell J S 1964 Physics 1195

[4] Werner R F 1989 Phys. Rev. A 404277
[5] Horodecki R, Horodecki P, Horodecki M and Horodecki K 2009 Rev. Mod. Phys. 81865

[6] Horodecki M, Horodecki P and Horodecki R 1996 Phys. Lett. A 2231

[7] Nielsen M A and Chuang I L 2000 Quantum Computation and Quantum Information (Cambridge: Cambridge University Press)

[8] Hanson R, Kouwenhoven L P, Petta J R, Tarucha S and Vandersypen L M K 2007 Rev. Mod. Phys. 791217

[9] Wendin G and Shumeiko V S 2006 Handbook of Theoretical and Computational Nanotechnology ed M Rieth and W Schommers (Los Angeles: ASP) pp 223-309 (see also arXiv:cond-mat/0508729)

[10] Hanson R and Awschalom D D 2008 Nature 4531043

[11] See e.g. Engel H-A and Loss D 2002 Phys. Rev. B 65195321

[12] Terhal B M 2000 Phys. Lett. A 271319

[13] Lewenstein M, Kraus B, Cirac J I and Horodecki P 2000 Phys. Rev. A 62052310

Terhal B M 2000 Phys. Lett. A 271319

[14] Gühne O, Hyllus P, Bruß D, Ekert A, Lewenstein M, Macchiavello C and Sanpera A 2002 Phys. Rev. A 66062305

[15] Gühne O, Hyllus P, Bruss D, Ekert A, Lewenstein M, Macchiavello C and Sanpera A 2003 J. Mod. Opt. 501079

[16] Gühne O and Tóth G 2009 Phys. Rep. 4741

[17] Bourennane M, Eibl M, Kurtsiefer C, Gaertner S, Weinfurter H, Gühne O, Hyllus P, Bruß D, Lewenstein M and Sanpera A 2004 Phys. Rev. Lett. 92087902

[18] Lu C-Y, Zhou X-Q, Gühne O, Gao W-B, Zhang J, Yuan Z-S, Goebel A, Yang T and Pan J-W 2007 Nat. Phys. 391

[19] Leibfried D et al 2005 Nature 438639 Häffner H et al 2005 Nature 438643

[20] Blaauboer M and DiVincenzo D P 2005 Phys. Rev. Lett. 95160402

Faoro L and Taddei F 2007 Phys. Rev. B 75165327

[21] Schlosshauer M 2005 Rev. Mod. Phys. 761267

[22] Loss D and DiVincenzo D P 1998 Phys. Rev. A 57120

[23] Petta J R et al 2005 Science 3092180

[24] Koppens F H L, Buizert C, Tielrooij K J, Vink I T, Nowack K C, Meunier T, Kouwenhoven L P and Vandersypen L M K 2006 Nature 442766

[25] Nowack K C et al 2007 Science 3181430

[26] Khaetskii A V and Nazarov Yu V 2001 Phys. Rev. B 64125316

[27] Zumbühl D M, Miller J B, Marcus C M, Campman K and Gossard A C 2002 Phys. Rev. Lett. 89276803

Miller J B, Zumbühl D M, Marcus C M, Lyanda-Geller Y B, Goldhaber-Gordon G, Campman K and Gossard A C 2003 Phys. Rev. Lett. 90076807

[28] Golovach V N, Khaetskii A and Loss D 2004 Phys. Rev. Lett. 93016601

[29] Abragam A 1961 The Principles of Nuclear Magnetism (Oxford: Oxford University Press)

[30] Paget D, Lampel G, Sapoval B and Safarov V I 1977 Phys. Rev. B 155780

[31] Khaetskii A V, Loss D and Glazman L 2002 Phys. Rev. Lett. 88186802

[32] Merkulov I A, Efros A L and Rosen M 2002 Phys. Rev. B 65205309

[33] Braun P-F et al 2005 Phys. Rev. Lett. 94116601

Johnson A C, Petta J R, Taylor J M, Yacoby A, Lukin M D, Marcus C M, Hanson M P and Gossard A C 2005 Nature 435925

Koppens F H L, Folk J A, Elzerman J M, Hanson R, Willems van Beveren L H, Vink I T, Tranitz H P, Wegscheider W, Kouwenhoven L P and Vandersypen L M K 2005 Science $\mathbf{3 0 9} 1346$

Taylor J M et al 2007 Phys. Rev. B 76035315

[34] Hanson R, Witkamp B, Vandersypen L M K, Willems van Beveren L H, Elzerman J M and Kouwenhoven L P 2003 Phys. Rev. Lett. 91196802 
[35] Elzerman J M, Hanson R, Willems van Beveren L H, Witkamp B, Vandersypen L M K and Kouwenhoven L P 2004 Nature 430431

[36] Shulman R G, Wyluda B J and Hrostowski H J 1958 Phys. Rev. 109808

[37] Hüttel A K, Weber J, Holleitner A W, Weinmann D, Eberl K and Blick R H 2004 Phys. Rev. B 69073302

[38] Kikkawa J M and Awschalom D D 1998 Phys. Rev. Lett. 804313

[39] Coish W A and Loss D 2004 Phys. Rev. B 70195340

[40] Chirolli L and Burkard G 2008 Adv. Phys. 57225 (see also arXiv:0809.4716)

[41] Herzog B and Hahn E L 1956 Phys. Rev. 103148

[42] Lindblad G 1976 Commun. Math. Phys. 48119

[43] Brandão F G S L 2005 Phys. Rev. A 72022310

Cavalcanti D and Terra Cunha M O 2006 Appl. Phys. Lett. 89084102

Gühne O, Reimpell M and Werner R F 2007 Phys. Rev. Lett. 98110502

Eisert J, Brandão F and Audenaert K 2007 New J. Phys. 946

[44] Peres A 1996 Phys. Rev. Lett. 771413

Horodecki M, Horodecki P and Horodecki R 1996 Phys. Lett. A 2231

[45] Tóth G and Gühne O 2005 Phys. Rev. Lett. 94060501

[46] Hanson R, Willems van Beveren L H, Vink I T, Elzerman J M, Naber W J M, Koppens F H L, Kouwenhoven L P and Vandersypen L M K 2005 Phys. Rev. Lett. 94196802

[47] Gühne O and Hyllus P 2003 Int. J. Theor. Phys. 421001

[48] Dür W, Vidal G and Cirac J I 2000 Phys. Rev. A 62062314

[49] Acín A, Bruß D, Lewenstein M and Sanpera A 2001 Phys. Rev. Lett. 87040401

[50] Greenberger D M, Horne M A and Zeilinger A 1989 Bell's Theorem, Quantum Theory, and Conceptions of the Universe (Dordrecht: Kluwer) pp 73-76 (see also arXiv:0712.0921)
Greenberger D M, Horne M A, Shimony A and Zeilinger A 1990 Am. J. Phys. 58 1131-43

[51] Verstraete F, Dehaene J, De Moor B and Verschelde H 2002 Phys. Rev. A 65052112

Lamata L, León J, Salgado D and Solano E 2007 Phys. Rev. A 75022318

[52] Kiesel N, Schmid C, Tóth G, Solano E and Weinfurter H 2007 Phys. Rev. Lett. 98063604

[53] Gühne O, Bodoky F and Blaauboer M 2008 Phys. Rev. A 78 060301(R)

[54] Simon C and Kempe J 2002 Phys. Rev. A 65052327

Dür W and Briegel H J 2004 Phys. Rev. Lett. 92180403

Aolita L, Chaves R, Cavalcanti D, Acín A and Davidovich L 2008 Phys. Rev. Lett. 100080501

Borras A, Majtey A P, Plastino A R, Casas M and Plastino A 2009 Phys. Rev. A 79022108

[55] Raussendorf R and Briegel H-J 2001 Phys. Rev. Lett. 865188

Raussendorf R, Browne D E and Briegel H-J 2003 Phys. Rev. A 68022312

[56] Hein M, Dür W, Eisert J, Raussendorf R, Van den Nest M and Briegel H-J 2005 Proc. Int. School of Physics Enrico Fermi on Quantum Computers, Algorithms and Chaos (Varenna, Italy, 2005)

Hein M, Dür W, Eisert J, Raussendorf R, Van den Nest M and Briegel H-J 2005 Proc. Int. School of Physics Enrico Fermi on Quantum Computers, Algorithms and Chaos ed G Casati, D L Shepelygansky, P Zoller and G Benenti (Varenna: IOS Press) pp 115-218 (see also arXiv:quant-ph/0602096)

[57] Dicke R H 1954 Phys. Rev. 9399

[58] Tóth G 2007 J. Opt. Soc. Am. B 24275

[59] See (2) in Briegel H-J and Raussendorf R 2001 Phys. Rev. Lett. 86910

[60] Bodoky F and Blaauboer M 2007 Phys. Rev. A 76052309 